This item was submitted to Loughborough's Research Repository by the author.

Items in Figshare are protected by copyright, with all rights reserved, unless otherwise indicated.

\title{
A generic electrical circuit for performance analysis of the fuel cell cathode catalyst layer through electrochemical impedance spectroscopy
}

PLEASE CITE THE PUBLISHED VERSION

http://dx.doi.org/10.1016/j.jelechem.2013.01.037

\section{PUBLISHER}

(C) Elsevier

VERSION

SMUR (Submitted Manuscript Under Review)

\section{PUBLISHER STATEMENT}

This work is made available according to the conditions of the Creative Commons Attribution-NonCommercialNoDerivatives 4.0 International (CC BY-NC-ND 4.0) licence. Full details of this licence are available at: https://creativecommons.org/licenses/by-nc-nd/4.0/

\section{LICENCE}

CC BY-NC-ND 4.0

\section{REPOSITORY RECORD}

Cruz-Manzo, Samuel, and Rui Chen. 2014. "A Generic Electrical Circuit for Performance Analysis of the Fuel Cell Cathode Catalyst Layer Through Electrochemical Impedance Spectroscopy". figshare. https://hdl.handle.net/2134/15609. 


\title{
A Generic Electrical Circuit for Performance Analysis of the Fuel Cell Cathode
}

\section{Catalyst Layer through Electrochemical Impedance Spectroscopy}

\author{
Samuel Cruz-Manzo and Rui Chen
}

Department of Aeronautical and Automotive Engineering, Loughborough University, UK

\section{Correspondence author's current contact information:}

Professor Rui Chen

Department of Aeronautical and Automotive Engineering

Loughborough University

Loughborough,

Leicestershire, LE11 3TU,

UK

Tel: +44(0)1509-227255

Fax: +44(0)1509-227275

e-mail: R.Chen@lboro.ac.uk 


\begin{abstract}
In this study, a generic electrical circuit is presented to characterise the frequency response of the Polymer Electrolyte Fuel Cell (PEFC) Cathode Catalyst Layer (CCL) at different current densities. The new electrical circuit is derived from fundamental electrochemical and diffusion theory. It consists of a transmission line in combination with distributed Warburg elements. The validation of this study is divided into a theoretical validation and an experimental validation. In the theoretical validation the impedance response of the CCL generated from three different circuits reported in the literature was compared with the simulated data from the generic electrical circuit. In the experimental validation, Electrochemical Impedance Spectroscopy (EIS) measurements were carried out in an $\mathrm{H}_{2} /$ air PEFC and through a three-electrode configuration in the measurement system and were compared with the simulated data from the generic circuit. The results show that the generic circuit is able to accurately reproduce the measured data of the CCL at different current densities and is able to represent the electrochemical and diffusion mechanisms of the CCL in the frequency domain. It is possible to generate a deeper understanding of how and where the chemical energy that is released from the redox reaction is being dissipated and retained within the real physical system.
\end{abstract}

Keywords: Electrochemical Impedance Spectroscopy, Cathode Catalyst Layer, Equivalent Circuit, Impedance Model, Electrochemical Mechanisms.

\title{
1. Introduction
}

Electrochemical Impedance Spectroscopy (EIS) is an experimental technique to measure the opposition to the flow of an Alternating Current (AC) within an electrochemical system which contains elements that dissipate energy and store energy. The opposition to the AC load, or impedance, can be measured over a range of frequencies, thereby revealing the frequency response of the system. One key advantage of the EIS technique is that it is non-invasive and can be applied insitu. Another advantage is that the frequency response tests are simple to carry out and can be easily tuned for greater accurately by using readily-available sinusoidal generators and precise measuring equipment. Frequency response is often represented in a complex-impedance-plane or Nyquist format. 
The data are presented as a locus of points, where each data corresponds to a different measurement frequency. Complex-impedance-plane plots are very popular because the locus of the points yields an insight into the possible mechanisms of governing phenomena. The frequency response of a Polymer Electrolyte Fuel Cell (PEFC) that results from EIS is in essence characterised by energy dissipating and energy storing elements of the cell. It can be represented by an equivalent electrical circuit model that is composed of resistors and capacitors respectively. By understanding the arrangement and magnitude of the resistive and capacitive elements in the equivalent circuit, it is possible to generate a deeper understanding of how and where the chemical energy that is released from the redox reaction is being dissipated and retained within the real physical system.

Different arrangements of electrical circuits from simple components [1,2,3,4] to more complicated transmission line representations $[5,6,7,8,9,10]$ have been reported in the literature to model and characterise different processes in a PEFC. Each equivalent electrical circuit can represent a specific physical process occurring in a PEFC. However the circuits are limited to a certain range of operating currents. The interpretation of the electrochemical mechanisms which are truly occurring in the PEFC using EIS will only be possible through a generic but robust equivalent circuit design which is derived from fundamental electrochemical and diffusion theory. In this study, a generic equivalent electrical circuit which is capable of characterising the impedance response of the Cathode Catalyst Layer (CCL) operated at any current of the polarisation curve has been developed. This new equivalent circuit is derived from fundamental electrochemical and diffusion theory. However, this new circuit has limitations in reproducing EIS measurements in the positive imaginary part of the compleximpedance-plane at low frequencies, which normally accounted for CO poisoning or adsorbed species in the Catalyst Layer (CL).

\section{Available Equivalent Circuits and Limitations}

The use of equivalent electrical circuits with the experimental EIS technique is a well-established methodology to characterise processes in the PEFC. Equivalent electrical circuits to interpret the mechanisms related to the impedance response of an electrochemical system have been used for over 
thirty years [11]. An electrical circuit can represent an identical impedance response to that obtained from the electrochemical system studied. Each electrical component in the electrical circuit describes a physical process that takes place in the electrochemical system. The most common electrical components to represent the impedance response in an electrochemical system are as follow:

Resistor, this component represents energy losses, electronic and ionic conductance in solid and aqueous medium. This element does not depend upon the frequency range applied.

Inductance, this component relates a magnetic field generated in electrical conductors of the measurement system and in the electrodes of an electrochemical system studied.

Capacitance, this component reflects the accumulation of electrostatic energy between two dissimilar materials.

Warburg, this element represents the opposition for diffusion of a chemical specie in a finite or semi-infinite planar medium in the frequency domain.

Constant Phase Element (CPE), this element reflects the exponential distribution of time-constants in an electrode-electrolyte interface.

Different arrangements of the electrical components have been reported in the literature to model and characterise other phenomenological processes in the PEFC. For instance, an electrical circuit proposed by Wagner [1] represents the PEFC during normal operation and during CO poisoning in the anode electrode. Ciureanu [12] proposed an electrical circuit to study the performance of the anode electrode of an $\mathrm{H}_{2} / \mathrm{H}_{2}$ fed Fuel Cell, this circuit also models the effect of $\mathrm{CO}$ poisoning and adsorbed species in the CL.

Randles circuit. The equivalent circuit for PEFC impedance generated from EIS analysis has typically been reported $[3,13]$ to be based on Randles circuits which are connected in series with a resistance. Each Randles circuit corresponds to an electrode (anode or cathode) while the resistance corresponds mainly to the polymer electrolyte membrane (PEM). In the case of the electrode, the 
electrical components of Randles circuit reflect; (i) a double-layer capacitance for the interface between the dissimilar materials, i.e., the nafion/carbon interface, and; (ii) a parallel resistance to charge transfer across the same interface, as shown in Fig.1a. The Randles circuit does not take into account the ionic resistance in the CL electrolyte and reactant transport limitations; therefore it can only be applied at low currents.

Randles circuit with Warburg element. At high currents, the product water formed in the CCL can begin to saturate the tortuous pathways of the porous network, which then acts as a resistance to the mass transport of oxygen from the interface with the Gas Diffusion Layer (GDL) to the catalyst sites in the CL. A Warburg element which describes resistance of diffusion of chemical species through a finite diffusion medium at the frequency domain and derived from Fick's second law and Faraday's Law has been broadly used together with the Randles circuit configuration to account for oxygen transport limitations at high currents in EIS measurements [2,4] as shown in Fig. 1b. This configuration does not take into account the ionic resistance in the CL electrolyte.

Transmission line circuit (no oxygen transport limitations). Eikerling and Kornyshev [5] proposed an electrical circuit using a transmission line to represent the impedance and to characterise the porous CL of PEFC's. This equivalent circuit consists of an upper rail to account for the ionic conduction, and a lower rail to represents the electron access in the CL. In the upper rail distributed resistances to account for the ionic resistance in the CL electrolyte are considered. Usually the distributed electron resistances are neglected to simplify the mathematical analysis because the resistance to ion transfer in the electrolyte of the CL is much greater than the resistance to the electron transfer in the carbon of the CL by several orders of magnitude. Between the upper rail and lower rail parallel distributed resistances and capacitances to account for the charge transfer process and the capacitance effect between the nafion/carbon interface are considered. These three elements are then repeated a finite number of times as shown in Fig. 1c. The transmission line circuit reported by Eikerling and Kornyshev to characterise the CCL operated at high currents cannot be used because it does not account for oxygen transport limitations in the CCL. At low currents, this phenomenon does not occur 
because the rate of water production is low and so does not appear as an equivalent electrical component in the transmission line.

\section{Fundamentals of CCL}

The catalyst layer is commonly formed by a composite structure of a matrix of carbon grains providing the electron conductivity, Pt supported on carbon as the catalyst, Teflon (PTFE) as a binder stabilizing the carbon matrix and as a hydrophobizing agent, and electrolyte network of perfluorosulfonate ionomer (PFSI) soaked with water. The carbon particles typically support noble metal or noble metal alloy deposits (i.e., platinum Pt, platinum-ruthenium PtRu) with sizes in the range of 2-5 $\mathrm{nm}$ on its surface to drive the electrochemical reactions forward at the operating temperatures of the PEFC. In addition, the catalyst layer contains a dispersion of polymer electrolyte to ensure continuity for ion conduction via the PEM. The resulting matrix structure is porous in nature and characterised by tortuous pathways for reactant transport. The matrix of carbon grains forms agglomerate structures. The agglomerated structure of the catalyst layer presents a bi-functional pore distribution. Primary pores are called to the pores existing inside the agglomerates between the $\mathrm{Pt} / \mathrm{C}$ particles. It has been proposed that the molecules of the polymer electrolyte do not penetrate into the Pt/C particles [14]. Instead these molecules form a pathway of ion conduction attached to the surface of the agglomerated structure. Secondary pores constitute the void spaces between agglomerates.

\subsection{Oxygen diffusion in the CCL}

- There are three modes of transport of oxygen in the CCL which can have an effect on mass transport limitations: gas-diffusion in the electrode-pore, dissolved oxygen diffusion in the liquid water surrounding the agglomerate, and dissolved oxygen in the ionomer phase [2].

- The finite diffusion distance for oxygen to reach the reaction sites in the CCL forms a complicated network of multi-phase parallel and serial paths [15]. 
- The finite diffusion distance could change in dimension for different CCL composition (e.g. nafion loading, porosity, tortuosity) and at different fuel cell operating conditions (current density, temperature, relative humidity, etc.) [16].

- The study of mass transport in the CCL is very complex; the literature has treated it with some simplifications and approximations [17].

\subsection{Performance}

In the literature [18] the performance of the CCL has been described by the following system of equations:

\section{Charge conservation:}

$C_{d l} \frac{\partial \eta}{\partial t}+\frac{\partial j}{\partial x}=-2 j_{o} \frac{c_{O}}{C_{O}^{*}} \sinh \left(\frac{\eta}{b}\right)$

Eq. 1 represents the current density $j$ across the thickness $x$ of the CCL that can take in the charge transfer due to the oxygen reduction reaction (ORR) or contribute to the charge in the capacitive effect between the dissimilar materials, i.e., the nafion-carbon interface; where $j_{o}$ represents the exchange current density, $b$ is the Tafel slope, $c_{o}$ represents the local oxygen concentration in the CCL, $c_{o}^{*}$ is the equilibrium oxygen concentration, $\eta$ is the CCL overpotential and $C_{d l}$ is the double layer capacitance.

Ohm's Law:

$j=-\sigma_{P} \frac{\partial \eta}{\partial x}$

Eq. 2 represents the potential in the electrolyte network of the CCL where $\sigma_{P}$ is the conductivity of ions in the electrolytic phase of the CCL.

Mass conservation:

$\frac{\partial c_{O}}{\partial t}-D \frac{\partial^{2} c_{O}}{\partial x^{2}}=-\frac{2 j_{o}}{z F} \frac{c_{O}}{c_{O}^{*}} \sinh \left(\frac{\eta}{b}\right)$ 
Eq. 3 represents the oxygen transport through the CCL thickness $x$ during the ORR where $D$ is the effective oxygen diffusion, $z$ the number of electrons consumed during the ORR and $F$ is the Faraday constant.

In the recent work of Kulykovsky [18] the above equations (Eqs. 1, 2 and 3) were combined to define the impedance response of the CCL. This resulted in complicated mathematical equations, which can only be solved numerically using mathematical software, and only approximate analytical solutions were reported. The author concluded that due to the thin CCL in PEFCs oxygen transport is usually good, therefore the resulting equation in the frequency domain from the combination of Eqs. 1, 2 and 3 could be simplified and used for fitting experimental EIS; however no attempt was made to validate the resulting CCL impedance model with real-world EIS measurements. It is worth mentioning that oxygen transport limitations in the CCL of PEFCs should not be considered as negligible because it is suspected that the CCL becomes flooded before the GDL because water is generated in the CCL and transported into the GDL, and has a lower porosity and smaller pore size, and tends to have higher flooding levels than the GDL [19].

Eq. 3 does not account for the oxygen diffusion on every single phase of the CCL material which can have an effect on mass transport limitations. As mentioned previously the finite diffusion distance for oxygen to reach the catalyst sites forms a complicated network of multi-phase parallel and serial paths and could change at different PEFC operation. A modelling approach to consider oxygen transport through the CCL thickness as part of a multi-species mixture using mass transport theory with concentrated solution theory [20] could give a detailed characterisation of the local oxygen concentration $c_{O}$ through the thickness $\mathrm{x}$ of the CCL during the ORR. Nevertheless the validation and application of the resulting equations with real-world EIS measurements could become a challenge.

\section{Development of a Generic Equivalent Circuit for CCL Analysis}

As reported by Brett et al. [21], the problem when choosing an equivalent electrical circuit is that must suit the bulk EIS results when actually different parts in the PEFC frequency response may be fit 
to the equivalent circuit with very different parameters or may require a different equivalent circuit altogether. An improved generic circuit design which is capable of interoperating variations in PEFC impedance characteristics is needed. The solution of the system of differential equations defining the physics of the CCL (Eq. 1, Eq. 2, Eq. 3) can be simplified to simulate the CCL impedance response by transforming them in the frequency domain and addressing considerations based on the theory of EIS.

\subsection{Modelling Considerations based on EIS}

- The use of a low amplitude perturbation in the EIS technique allows the use of a linear model to interpret the impedance response of the CCL from EIS.

- EIS measurements are carried out in PEFCs operated at steady state; therefore it is possible to relate the current density from Faraday's Law (oxygen consumed in the ORR) with diffusion flux from Fick’s Law.

- EIS only measures bulk parameters in the total CCL thickness and reflects a total mass transport resistance and a total finite diffusion distance for the three modes of oxygen transport in the CCL.

- In the mathematical treatment of this study, the finite diffusion distance $y$ for oxygen to diffuse through the CCL will be considered to be independent from the thickness $x$ of the CCL, as shown in Fig. 2, to simulate the CCL impedance response.

- The change in oxygen concentration in the CCL during the ORR and represented in EIS measurements will be considered from the CCL-GDL interface $c_{o}^{*}$ at $y=0$ to the CCL-PEM interface $c_{o}^{\prime}$ at $y=\delta$, as shown in Fig. 2.

- Even though this mathematical treatment results in an over-simplification of the oxygen diffusion in the total CCL this will simplify the mathematical analysis, and therefore the resulting model will present parameters commonly known in the electrochemical area such as the Warburg impedance [4] which allows the characterisation of the low frequency semicircle of EIS measurements accounted to oxygen transport limitations. 


\subsection{Performance in the Frequency Domain}

A new system of equations in the frequency domain can be defined based on the modelling considerations addressed in section 4.1 and EIS theory as such:

Charge conservation equation at frequency domain. Considering trigonometric identities in Eq. 1 yields:

$\frac{\partial j}{\partial x}=-j_{o} \frac{C_{O}}{c_{O}^{*}}\left[\exp \left(\frac{\eta}{b}\right)-\exp \left(-\frac{\eta}{b}\right)\right]-C_{d l} \frac{\partial \eta}{\partial t}$

Eq. 4 expresses the charge distribution in the CCL thickness $x$ considering boundary conditions as CCL-PEM interface at $x=0$ and GDL-CCL at $x=1$ [18]. The mathematical treatment in this study will consider the boundary conditions shown in Fig. 2 as: GDL-CCL at $x=0$ and CCL-PEM at $x=1$, therefore a change in sign (positive) has to be considered on the right-hand side of Eq. 4. The first exponent on the right-hand side of Eq. 4 represents the ORR and the second exponent represents the reverse reaction of the ORR. Also when the overpotential $\eta$ increases during CCL operation the contribution of the second exponent becomes small, and therefore it can be neglected, as such:

$\frac{\partial j}{\partial x}=j_{o} \frac{c_{O}}{c_{O}^{*}} \exp \left(\frac{\eta}{b}\right)+C_{d l} \frac{\partial \eta}{\partial t}$

EIS technique allows the use of a linear equation to simulate impedance spectra. A linear model can be derived using the Taylor series expansion to Eq. 5, (Appendix A) as such:

$\frac{\partial \bar{j}}{\partial x}=\frac{C_{O}}{C_{O}^{*}} \frac{\bar{\eta}}{R_{C}}+C_{d l} \frac{\partial \bar{\eta}}{\partial t}$

where $R_{C}$ represents the charge transfer resistance during the ORR and is defined as $R_{C}=b / j_{0} \exp \left(\eta_{S} / b\right), \eta_{S}$ represents the activation overpotential in CCL and $c_{c_{O}}$ represents the local oxygen concentration in the CCL. This local concentration will depend on the diffusion of oxygen through the multiphase parallel and serial paths in the CCL. To simplify the mathematical treatment, the ratio between oxygen concentration at the CCL-PEM interface and CCL-GDL interface $c_{o}^{\prime} / c_{0}^{*}$ defined in previous section 4.1 is taking into account in Eq. 6. This ratio between oxygen concentrations is considered from the fact that EIS technique only measures the change in oxygen 
concentration in the total CCL thickness. This mathematical treatment will allow the derivation of the Warburg Impedance which has been broadly used in the EIS area [2,4] to characterise oxygen transport limitations during PEFC operation. The electrochemical reaction in the PEFC results in an inhomogeneous distribution of charge in the CCL. To correct for this inhomogeneity, a constant phase element (CPE), $Y s^{P}$ with $s=i \omega$, which is defined in the frequency domain has to be used in the Laplace transform $s$ of Eq. 6 to replace the capacitor $C_{d l}$ [22], as such:

$\frac{\partial \bar{j}(s)}{\partial x}=\frac{c_{O}^{\prime}(s)}{c_{O}^{*}(s)} \frac{\bar{\eta}(s)}{R_{C}}+Y s^{P} \bar{\eta}(s)$

where $s=i \omega$ is the frequency domain defined from Laplace domain, $\omega$ is the angular frequency, $\boldsymbol{i}$ is the imaginary component, $c_{o}^{\prime}(s)$ is the oxygen concentration at the CCL-PEM interface, $c_{o}^{*}(s)$ is the equilibrium oxygen concentration at the GDL-CCL interface in the frequency domain, $Y$ represents a parameter related to $\mathrm{CPE}$, superscript $P$ represents a parameter to correct the inhomogeneity in the distribution of charge, $x$ represents the thickness (dimensionless) of the CCL from $x=0$ CCL-GDL interface to $x=1$ CCL-PEM interface.

Oxygen Transport during the ORR at frequency domain. The concentration profile of chemical species during a simple electron-transfer reaction $\mathrm{Ox}+e^{-} \stackrel{K}{\leftrightarrow}$ Re can be derived from the general theory of controlled-current methods [23]. This theory solves Fick’s Second Law of diffusion and relates its solution with the flux of chemical species and Faraday's Law to account for the change in concentration of chemical species during an electrochemical reaction at a fixed current. The same procedure will be taken into account for the derivation of the change of oxygen concentration during the ORR in the CCL.

The first term on the right-hand side of Eq. 7 represents the current at which oxygen is consumed during the ORR in the frequency domain:

$$
\frac{\partial \bar{j}_{1}(s)}{\partial x}=\frac{c_{O}^{\prime}(s)}{c_{O}^{*}(s)} \frac{\bar{\eta}_{1}(s)}{R_{C}}
$$


From Faraday's Law it is possible to establish that the current density in the frequency domain is proportional to the charge transferred and the consumption of reactant:

$\frac{\partial \bar{j}_{1}(s)}{\partial x}=z F v(s)$

where $\mathrm{z}$ is the number of electrons consumed during the ORR and $\mathrm{F}$ is the Faraday constant. From Fick's First Law it is possible to establish that the flux of reactant is proportional to concentration gradient:

$$
v(s)=-D{\frac{\partial c_{o}(y, s)}{\partial y}}_{y=\delta}
$$

where $D$ is the effective diffusion coefficient of oxygen and $y$ is the finite distance for oxygen to diffuse in the CCL, as shown in Fig. 2. In steady state the current density at which the oxygen is consumed in the ORR from Faraday's Law is equal to the diffusion flux from Fick's First Law [16]. Combining Eqs. 8, 9 and 10 yields:

$\frac{c_{O}^{\prime}(s)}{c_{O}^{*}(s)} \frac{\bar{\eta}_{1}(s)}{z F R_{C}}=-D \frac{\partial c_{O}(y, s)}{\partial y} \quad{ }_{y=\delta}$

EIS measurements are carried out in PEFCs operated at steady state. At steady state the oxygen concentration is independent of time; hence the Fick's Second Law can be expressed in Laplace domain s, as such:

$D \frac{\partial^{2} c_{O}(y, s)}{\partial y^{2}}=s c_{O}(y, s)-c_{O}^{*}$

Eq. 12 represents the Fick's Second Law in the Laplace domain considering the initial condition at $t=0$ as $c_{O}(y, 0)=c_{O}^{*}$, The solution of Eq. 12 through the method of undetermined coefficients for a nonhomogeneous linear differential equation and considering boundary conditions from CCL-GDL interface $c_{O}(0, s)=c_{O}^{*}(s)$ to CCL-PEM interface $c_{O}(\delta, s)=c_{O}^{\prime}(s)$, as shown in Fig. 2, yields:

$c_{O}(y, s)-c_{O}^{*}(s)=\frac{\left[c_{O}^{*}(s)-c_{O}^{\prime}(s)\right] \exp \left(\lambda_{1} y\right)}{\exp \left(\lambda_{2} \delta\right)-\exp \left(\lambda_{1} \delta\right)}+\frac{\left[c_{O}^{\prime}(s)-c_{O}^{*}(s)\right] \exp \left(\lambda_{2} y\right)}{\exp \left(\lambda_{2} \delta\right)-\exp \left(\lambda_{1} \delta\right)}$

where $\lambda_{1,2}= \pm \sqrt{s / D}$. The ratio between oxygen concentration at the CCL-PEM interface and CCLGDL interface $c_{o}^{\prime}(s) / c_{o}^{*}(s)$ can be expressed as a function of mass transport resistance in the frequency 
domain and charge transfer resistance. Differentiating Eq. 13 with respect to $y$ and substituting it into Eq. 11 and considering trigonometric identities, the Laplace form $\bar{\eta}_{1}(s)=\bar{n}_{1} / s$ and $c_{O}^{*}(s)=c_{O}^{*} / s$, and replacing $\quad \bar{\eta}_{1}$ by $\bar{\eta}_{1}=R T$ / zF which represents a linearized relation of the overpotential [23], where $R$ is the ideal gas constant and $T$ is the operating temperature, yields :

$\frac{c_{O}^{\prime}(s)}{c_{O}^{*}(s)}=\frac{R_{C}}{R_{C}+Z_{W}}$

where

$Z_{W}=R_{W} \frac{\tanh \left(s T_{W}\right)^{0.5}}{\left(s T_{W}\right)^{0.5}}$

is known as the Warburg Impedance [2,4] and represents the mass transport resistance in the frequency domain and simulates the low frequency semicircle in EIS measurements of PEFCs,

with

$R_{W}=\frac{R T \delta}{Z^{2} F^{2} C_{O}^{*} D}$

defined as resistance for the diffusion process and

$T_{W}=\frac{\delta^{2}}{D}$

defined as the time constant to diffuse oxygen through the CCL.

Ohmic Law at frequency domain. The ion conduction in the CCL depends upon the electrolytic dispersion and the state of hydration within the CCL. It is assumed that the resistance to the electron flow in the electrode network is smaller than the ionic resistance in the electrolyte network. Therefore, electronic ohmic loss in CCL can be regarded as being negligible $[6,8,9]$. The potential in the electrolyte network can be expressed by Ohms law:

$$
\frac{\partial \bar{\eta}(s)}{\partial x}=\bar{j}(s) R_{P}
$$

where $R_{P}$ is the resistance to the flow of ions in the electrolytic phase of the CCL. 


\subsection{Current Density in Frequency Domain}

Substituting Eq. 14 into Eq. 7 yields:

$\frac{\partial \bar{j}(s)}{\partial x}=\frac{\bar{\eta}(s)}{R_{C}+Z_{W}}+Y s^{P} \bar{\eta}(s)$

If Eq. 19 is substituted into Eq. 18 and considering the activation overpotential $\eta_{s}$ in $R_{C}$ as a constant due to $R_{P} / R_{C}<<1$ [6], (Appendix B), yields:

$\frac{\partial^{2} \bar{j}(s)}{\partial x^{2}}=R_{p}\left[\frac{\bar{j}(s)}{R_{C}+Z_{W}}+Y s^{P} \bar{j}(s)\right]$

Eq. 20 is a $2^{\text {nd }}$ order homogeneous equation and represents the current distribution through the thickness of the CCL in the frequency domain taking into account mass transport resistance, electrode kinetics, charge capacitance and ionic resistance in the CCL. Its solution can be obtained by applying the method of the nth-order homogeneous equation with constant coefficients:

$\bar{j}(s)=A \exp \left(\gamma_{1} x\right)+B \exp \left(\gamma_{2} x\right)$

where $\quad \gamma_{1,2}= \pm \sqrt{R_{p}\left[\frac{1}{R_{C}+Z_{W}}+Y s^{p}\right]}$ represents the distinct roots of the characteristic equation represented.

Evaluating boundary conditions in Eq. 21, at the GDL-CCL $x=0$ and $\bar{j}(s)=0$, while at the CCLPEM interface $x=1$ and $\bar{j}(s)=j_{m}(s)$, where $j_{m}(s)$ represents the current density of the cell, gives the current density in the frequency domain,

$$
\bar{j}(s)=j_{m}(s) \frac{\sinh \left(\gamma_{1} x\right)}{\sinh \left(\gamma_{1}\right)}
$$

\subsection{Overpotential in Frequency Domain}

The overpotential in the frequency domain can be obtained by arranging Eq. 7 as:

$$
\bar{\eta}(s)=\frac{R_{C}+Z_{W}}{1+Y s^{P}\left(R_{C}+Z_{W}\right)} \frac{\partial \bar{j}(s)}{\partial x}
$$


Differentiating Eq. 22 with respect to $x$ and substituting into Eq. 23 gives the overpotential in the frequency domain

$$
\bar{\eta}(s)=\frac{\left[R_{C}+Z_{W}\right] j_{m}(s) \gamma_{1} \cosh \left(\gamma_{1} x\right)}{\left\{1+Y s^{P}\left[R_{C}+Z_{W}\right]\right\} \sinh \left(\gamma_{1}\right)}
$$

\subsection{Impedance Model of CCL}

The impedance of the CCL is defined as the ratio between the overpotential, Eq. 24, and the current, Eq. 22 at frequency domain with $s=i \omega$.

$$
Z_{C C L}=\frac{\bar{\eta}(i \omega)}{\bar{j}(i \omega)}=\frac{\left[R_{C}+Z_{W}\right] \gamma_{1} \operatorname{coth}\left(\gamma_{1}\right)}{1+Y(i \omega)^{P}\left[R_{C}+Z_{W}\right]}
$$

With $\gamma_{1}=\sqrt{R_{p}\left[\frac{1}{R_{C}+Z_{W}}+Y(i \omega)^{P}\right]}$

Eq. 25 represents the opposition to the flow of an alternating current AC within the CCL which contains physical processes that dissipate energy and store energy. The opposition to the AC load, or impedance, can be measured over a range of frequencies, thereby revealing the frequency response of the CCL. By understanding the magnitude of the electrochemical and oxygen transport mechanisms represented in Eq. 25, it is possible to generate a deeper understanding of how and where the chemical energy that is released from the redox reaction is being dissipated and retained within the CCL. For the specific case where $Z_{w}$ is considered to be negligible either due to a high diffusion coefficient or high equilibrium oxygen concentration, Eq. 25 will represent the impedance response of the CCL with equilibrium boundary conditions in terms of oxygen concentration. These conditions can occur for low current operation and as such, Eq. 25 reduces to the equation representing a transmission line equivalent circuit as reported by Makharia et al. [6]. The solution of Eq. 25 over a range of frequencies will contain real and imaginary components, which can be presented on a Nyquist plot. Eq. 25 can be represented through the impedance of the electrical circuit shown in Fig. 3. The four elements represented in Fig. 3 are repeated $k_{N}$ a finite number of times, where k represents a collection of carbon-supported catalytic agglomerates coated by a thin layer of polymer electrolyte. Eq. 25 represents the impedance of the generic circuit considering $k_{N}$ parameters. 


\section{Model Validation}

In this study the validation of the impedance spectrum generated from the generic circuit (Eq. 25) is divided into a theoretical validation and an experimental validation. In the theoretical validation the impedance spectrum generated from the generic electrical circuit proposed in this study is validated against the CCL impedance response generated from three different electrical circuits commonly used in the literature. In the experimental validation the impedance spectrum generated from the generic circuit is compared with measured impedance data of an $\mathrm{H}_{2} /$ air PEFC operated at $8 \mathrm{~mA} / \mathrm{cm}^{2}$ and 0.12 $\mathrm{A} / \mathrm{cm}^{2}$.

\subsection{Theoretical validation}

Equivalent Circuits neglecting oxygen transport limitations. The impedance response of a $\mathrm{H}_{2} / \mathrm{air}$ PEFC was investigated by Ciureanu and Roberge [3]. The authors used a Randles electrical circuit as shown in Fig. 1a to characterise the CCL impedance response at low currents. This electrical circuit accounts just for the charge transfer resistance during the ORR and does not account for ionic resistance in the CCL electrolyte and oxygen transport limitations. The parameters from the Randles circuit as reported by Ciureanu such as charge transfer resistance $R_{C}=0.4148 \Omega . \mathrm{cm}^{2}$, and CPE derived from the capacitance $C=0.3950 \mathrm{~F} / \mathrm{cm}^{2}$ were substituted into Eq. 25 . The ionic resistance $R_{P}$ was considered with a small order of magnitude $10^{-9}$ to be regarded as being negligible. The Warburg impedance $Z_{W}$ was considered as being negligible $Z_{W}=0$ in Eq. 25. These two elements do not appear in the Randles configuration. The results in Fig. 4a show a good agreement between simulated spectrum using Eq. 25 and the measured data from the Randles circuit.

Makharia et al. [6] estimated the ionic resistance in the CCL for a $5 \mathrm{~cm}^{2}$ PEFC $\left(\mathrm{H}_{2}-\mathrm{O}_{2}\right)$ operated at low current with MEA 0.8 and 0.4 Nafion/Carbon (N/C) ratio through impedance measurements. The ionic resistance $R_{P}=0.103 \Omega . \mathrm{cm}^{2}$, charge transfer resistance $R_{C}=0.783 \Omega . \mathrm{cm}^{2}$ and capacitance $C=20 \mathrm{mF} . \mathrm{cm}^{-2}$ of the CCL were estimated by fitting the experimental PEFC spectrum to the equivalent electrical circuit type transmission line as shown in Fig. 1c using Zview software (Transmission Line- 
Open Circuit Terminus, DX-Type 6, Scribner Associates, Inc., version 2.3). This electrical circuit does not account for oxygen transport limitations in the CCL. The parameters of the transmission line were substituted into Eq. 25 to simulate the experimental impedance spectrum with no mass transport effect $Z_{W}=0$ as reported by Makharia et al. and as shown in Fig.4b. A $45^{\circ}$ region representing the ionic resistance in the CCL at high frequency is presented and has been estimated by projecting the $45^{\circ}$ region onto the real part Z' and calculated as $R_{P} / 3$. The diameter of the semicircle is related to the charge transfer resistance during the ORR.

Equivalent Circuit considering oxygen transport limitations. The parameters of the Randles circuit and Warburg element to characterise the impedance response of CCL operated at high currents were reported by Fouquet et al. [4]. This electrical circuit does not account for the ionic resistance in the CCL electrolyte. This electrical circuit can fit the low frequency semicircle in the impedance spectrum which normally accounted for mass transport limitations because of the shortage of the oxygen supplied during fuel cell operation. The charge transfer resistance $R_{C}=0.008 \Omega, C P E$ with $Y$ $=1.109 \mathrm{~S}^{\mathrm{P}} / \Omega$, and Warburg element with $R_{W}=0.0034 \Omega, T_{W}=0.0872 \mathrm{sec}$ were substituted in Eq. 25 . The ionic resistance $R_{P}$ was considered with a small order of magnitude $10^{-9}$ in Eq. 25 to be regarded as being negligible. The results show a good agreement between simulated spectrum using Eq. 25 and the generate data from the Randles-Warburg circuit, as shown in Fig. 5. The diameter of the semicircle at high frequencies is related to the charge transfer resistance during ORR. The semicircle at low frequencies is related to gas phase oxygen transport limitations in the CCL-GDL interface.

\subsection{Experimental validation}

Experimental. A $25 \mathrm{~cm}^{2}$ commercially-available fuel cell and test rig acquired from Baltic Fuel Cells were used for the experimental tests. The MEA consisted of a catalyst coated membrane DuPont Nafion-115 with a platinum loading of $0.4 \mathrm{mg} / \mathrm{cm}^{2}$ and carbon black for the electrodes. The thickness of the CCL is $12 \mu \mathrm{m}$. The contact pressure on the active cell area was adjusted through a pneumatic air cylinder from the Baltic Fuel Cell compression unit. The contact pressure on the active area was fixed 
to $1.4 \mathrm{~N} / \mathrm{mm}^{2}$. The operational temperature was $50^{\circ} \mathrm{C}$ and the back gas pressure was held to $0.9 \mathrm{bar}(\mathrm{g})$ for both the anode and cathode. Flow rates were held constant during all the experiments, hydrogen to the anode was supplied at a stoichiometry of 2 and air to the cathode supplied at a stoichiometry of 2.5. The PEFC was operated with $98 \%$ hydrogen relative humidity (RH) in the anode and 55\% RH in the cathode. EIS measurements were carried out through a Solartron 1280C electrochemical interface and a Solartron 1280C frequency response analyser as shown in Fig. 6. EIS measurements were carried out at two different current densities 0.008 and $0.12 \mathrm{~A} / \mathrm{cm}^{2}$. The frequency scan was performed from $20 \mathrm{kHz}$ down to $0.1 \mathrm{~Hz}$, with an alternating voltage signal and $10 \mathrm{mV}$ amplitude. To separate the impedance spectrum of the cathode from the impedance spectrum of the cell, a reference electrode made of a platinum wire was inserted such that it was in direct contact with the membrane of the cathode side. Under such conditions, the signals are measured between the working electrode (WE) and the reference electrode (RE), and the current induced is collected by the counter electrode (CE).

Measured Cathode Impedance Response. In these experimental results the use of a reference electrode in the measurement system ensures that the data accounting for the processes in the cathode are captured for analysis and interpretation. The resulting impedance is commonly shown in a complex plane and represents the electrochemical and diffusion mechanisms of the PEFC in the frequency domain. Fig. 7 shows the measured impedance response of the cathode obtained through a three-electrode configuration in the measurement system. The diameter of the spectrum decreases with increasing current density from $0.008 \mathrm{~A} / \mathrm{cm}^{2}$ to $0.12 \mathrm{~A} / \mathrm{cm}^{2}$. At $0.008 \mathrm{~A} / \mathrm{cm}^{2}$ the kinetics of the ORR dominates the cathode performance and the impedance spectrum mainly represents the charge transfer effect during the ORR. At $0.12 \mathrm{~A} / \mathrm{cm}^{2}$ the diameter of the spectrum decreases due to an increase in the driving force for the interfacial oxygen reduction process [24]. At current density of $0.12 \mathrm{~A} / \mathrm{cm}^{2}$ the presence of an overlapped second semicircle at low frequencies demonstrates that oxygen transport limitations becomes a limiting factor in the PEFC performance [3,24], as shown in Fig. 7a. Oxygen transport limitations are mainly attributed to high water concentration during the ORR which acts as a resistance for oxygen to permeate through the GDL and CCL. The hypothesis 
that there is an increase in water concentration in these EIS results is supported by the fact that at 0.12 $\mathrm{A} / \mathrm{cm}^{2}$ there is a decrease in ohmic resistance in the PEM. This effect is shown in Fig. $7 \mathrm{~b}$ where the impedance spectra intercept the real component Z' in the complex plot [3] at high frequencies. The measured data with positive imaginary components at high frequencies as shown in Fig. $7 \mathrm{~b}$ are related to the inductance of the electrical cables of the measurement system [25].

Validation at $8 \mathbf{~ m A} / \mathbf{c m}^{2}$. The product of water formed in the CCL can begin to saturate the tortuous pathways of the porous network, which then acts as a resistance to the mass transport of oxygen from the interface with GDL to the catalyst sites in the CCL. At low currents, this phenomenon does not occur because the rate of water production is low; therefore the mass transport resistance (Warburg impedance) is considered as being negligible $Z_{w}=0$ in Eq. 25, and the generic circuit shown in Fig. 3 accounted to the CCL takes the form as the circuit reported by Eikerling and Kornyshev [5] as shown in Fig. 1c. The generic circuit that represents the CCL with $Z_{W}=0$ is connected in series with an inductor element and a resistor as shown in Fig. 8 to simulate the cathode impedance response neglecting oxygen transport limitations and obtained through a three-electrode configuration in the measurement system. Therefore the impedance response of the cathode neglecting oxygen transport limitations and obtained from a three electrode configuration can be represented as:

$$
Z_{\text {Cathode }}=L(i \omega)+R_{e}+\frac{R_{C} \gamma_{1} \operatorname{coth}\left(\gamma_{1}\right)}{1+Y(i \omega)^{P} R_{C}}
$$

where $L$ represents the inductance in the electrical cables of the measurement system, $R_{e}$ represents the total ohmic resistance to flow electrons and ions in the bipolar plate, GDL and PEM and the third term on the right-hand side represents the CCL impedance (Eq. 25) neglecting oxygen transport limitations $Z_{W}=0$. The circuit shown in Fig. 8 was fitted to the measured impedance response at $8 \mathrm{~mA} / \mathrm{cm}^{2}$ using Zview software (Transmission Line-Open Circuit Terminus, DX-type 6, Scribner Associates, Inc., version 3.0). The parameters of the transmission line extracted from the measured data are shown in Table I. The parameters from Table I were substituted into Eq. 26 to simulate the cathode impedance response at $8 \mathrm{~mA} / \mathrm{cm}^{2}$. Figure 9 shows that with the parameters given in Table I, 
the theoretical model is capable of simulating the frequency response of the cathode impedance. The results show a good agreement between the measured and simulated data in the complex plot with an exception at the highest frequencies (EIS measurements with positive imaginary components Z'), as shown in Fig. 9. EIS measurements with positive imaginary components at the high frequency end of the spectrum have been mainly attributed to the inductance of the electrical cables of the measurement system [25]. In a previous study [26] it was demonstrated that the property of causality in the Kramers-Kroning mathematical relations for EIS measurements is violated by the external inductance of the measurement cables. Also it was demonstrated that the inductance of the measurement system deforms the high frequency region of the impedance spectrum and as a result it is possible to draw an incorrect conclusion about the electrochemical mechanisms at high frequencies by visual inspection, e.g. ohmic resistance. Therefore it is possible to conclude that the measured data with positive imaginary components do not represent the physics and chemistry of the cathode. A $45^{\circ}$ region at high frequencies is shown in Fig. 9b. This has been associated with the ionic resistance $R_{P}$ in the CCL electrolyte [6,7]. The semicircle of the spectrum is related to the charge transfer resistance $R_{C}$ during the ORR and charge capacitance between dissimilar materials, i.e. nafion-carbon interface.

Validation at $0.12 \mathrm{~A} / \mathbf{c m}^{2}$. At high currents, the product water formed in the CCL can begin to saturate the tortuous pathways of the porous network, which then acts as a resistance to the mass transport of oxygen from the interface with the GDL to the catalyst sites in the CCL. Under such conditions the electrical circuit shown in Fig. 3 is connected in series with an inductor element and a resistor as shown in Fig. 10 to simulate the cathode impedance response considering oxygen transport limitations and obtained through a three-electrode configuration in the measurement system. The impedance response of the cathode considering oxygen transport limitations and obtained from a three electrode configuration can be represented as:

$$
Z_{\text {Cathode }}=L(i \omega)+R_{e}+\frac{\left[R_{C}+Z_{W}\right] \gamma_{1} \operatorname{coth}\left(\gamma_{1}\right)}{1+Y(i \omega)^{P}\left[R_{C}+Z_{W}\right]}
$$

where the third term represents the impedance equation of the CCL derived in section 4.5 (Eq. 25). Some commercial software (Zview, ZMAN, etc.) fit experimental impedance data by using nonlinear 
regression strategies such as Levenberg-Marquardt, Gauss-Newton Method, etc., and with the use of available equivalent electrical circuits (Fig 1a-c). However this technique strongly requires some parameters of the electrical circuit to be defined as initial values in order to achieve the best-fit. The simulated data from the circuit shown in Fig. 10 were compared with the cathode measured data at $0.12 \mathrm{~A} / \mathrm{cm}^{2}$ using a graphical user interface (GUI) developed in Matlab ${ }^{\circledR}$, as shown in Fig. 11. The GUI allows the fitting of the parameters from Eq. 27 to achieve a good agreement between the experimental and simulated data. The least-squares fitting method was used in order to find the best fit between the model and the measured data. A good quality fit is obtained when the sum of the deviations squared (least-square error) between the simulated and measured impedance data as a minimum value, for example $<0.1$. The GUI developed for EIS analysis relies on the mathematical model of this study which is based on fundamental electrode and diffusion theory. The electrochemical and diffusion parameters defined in the model are related to one another in which counteracting interdependencies are accounted for, for instance, by changing the ionic resistance at high frequency, the values of the impedance spectrum at low frequency will be changed. Eq. 27 was fitted to the measured data at $0.12 \mathrm{~A} / \mathrm{cm}^{2}$ and the results are shown in Table II. The measured data with positive imaginary components are mainly attributed to the inductance of the measurement system as discussed in a previous study [26]. In the EIS results shown in Fig. $12 \mathrm{~b}$ a $45^{\circ}$ region can be noticeable in the high frequency region of the spectrum. This linear region has been associated with the ionic resistance in the CCL electrolyte [6,7]. The impedance results of Fig. 12 reflect the overlapping of two semicircles. One at high-medium frequencies is related to the charge transfer resistance during the ORR, and the other at low frequencies is related to gas phase oxygen transport limitations in the CCL-GDL interface [27]. It has been proposed [15] that improving one property of the CL can adversely affect another. For instance, by increasing the amount of the electrolyte between the agglomerates, the ionic conductivity can be enhanced but also closes void spaces for reactant transport. This effect could also be reflected in the impedance response of the CCL at high and low frequencies. 


\section{Discussion}

The electrochemical and diffusion mechanisms in the CCL change during fuel cell operation. The use of equivalent electrical circuits with the experimental EIS technique is a well-established methodology to characterise processes in the CCL. However the circuits reported in the literature are limited to a certain range of operating current densities and do not represent the electrochemical and diffusion processes which are truly occurring in the CCL. For example the ionic resistance in the CCL has been commonly estimated in EIS measurements using the transmission line circuit during CCL operation at low currents. However the ionic resistance in the CCL is also dependent on the hydrated state and water concentration of the CCL at high currents. If the ionic resistance in the CCL electrolyte (straight line in the CCL spectrum at high frequency) is reduced by increasing Nafion loading and water concentration, an increase in oxygen transport limitations (low frequency semicircle in the CCL spectrum) is expected. This study has demonstrated that the electrochemical and diffusion mechanisms of the CCL in the frequency domain can be simulated through a newlydeveloped equivalent circuit. This electrical circuit was derived from fundamental electrochemistry and diffusion theories. This new circuit has limitations in reproducing EIS measurements in the positive imaginary part of the complex-impedance-plane at low frequencies, which normally accounted for CO poisoning or adsorbed species in the CL. This is the aim of a future work.

\section{Conclusions}

A generic electrical circuit based on fundamental electrode and diffusion theory has been developed to characterise the impedance response of the CCL at different current densities. The current study begins by defining the equations of the CCL performance in the frequency domain from EIS such as charge conservation, oxygen concentration during the ORR and potential in the CCL electrolyte network. Modelling considerations based on EIS theory were taking into account to simplify the mathematical treatment in this study. EIS reflects only bulk measurements on the total CCL thickness, therefore the finite diffusion distance and surface concentration of oxygen in the CCL are considered to be independent from the thickness of the CCL. This approach simplifies the mathematical treatment to predict the impedance response of the CCL, and as result it can be successfully validated against 
real-world EIS measurements. The resulting theoretical model is validated against the impedance response generated from three electrical circuits reported in the literature. Also the theoretical model is validated against the measured EIS response of an $\mathrm{H}_{2} /$ air PEFC. The results show that the model can predict the impedance response of the CCL at different current densities of the polarisation curve. The model has established a wider scope to relate the measured electrochemical impedance data to the fundamental theory of PEFCs.

\section{Acknowledgments}

The authors thank the Mexican National Council for Science and Technology (CONACYT) for the sponsorship of the Ph.D research study of S. Cruz-Manzo (grant no. 183195).

\section{Appendix A}

Solution of Linear Equations approximated by the Taylor Series Expansion

$\frac{d \eta}{d t}=f(\eta, j)$

Expanding the second term of Eq. A-1 which contains the nonlinear term in Taylor series up to its first derivative form, gives:

$$
f(\eta, j) \approx f\left(\eta_{s}, j_{s}\right)+\left(\frac{\partial f}{\partial \eta}\right)_{n_{s}, j_{s}}\left(\eta-\eta_{s}\right)+\left(\frac{\partial f}{\partial j}\right)_{\eta_{s}, j_{s}}\left(j-j_{S}\right)
$$

Given that the expansion is carried out around a steady state $\left(\eta_{s}, j_{s}\right)$, Eq. (A-1) can be expressed as

$\frac{d \eta_{s}}{d t}=f\left(\eta_{s}, j_{s}\right)=0$

Since $\eta_{\mathrm{s}}$ is a constant, the left side of Eq. (A-1]) can be expressed as

$\frac{d \eta}{d t}=\frac{d\left(\eta-\eta_{s}\right)}{d t}=\frac{d \bar{\eta}}{d t}$

where $\bar{\eta}=\eta-\eta_{s}$ represents the deviation of variable $\eta$ in the steady state $\eta_{s}$. The linear equation can be defined as:

$\frac{d \bar{\eta}}{d t}=\left(\frac{\partial f}{\partial \eta}\right)_{\eta_{s}, j_{s}} \bar{\eta}+\left(\frac{\partial f}{\partial j}\right)_{\eta_{s}, j_{s}} \bar{j}$ 


\section{Appendix B}

Charge transfer resistance $R_{C}=b / j_{0} \exp \left(\eta_{S} / b\right) \quad$ overpotential in the electrolyte $\frac{d \eta_{S}}{d x}=R_{P} j$ $\frac{R_{P}}{R_{C}}=\frac{d \eta_{S}}{d x} \frac{j_{0} \exp \left(\eta_{S} / b\right)}{b j} \quad$ Separating variables $\quad \exp \left(\eta_{S} / b\right) d \eta_{S}=\frac{R_{P}}{R_{C}} \frac{b j}{j_{0}} d x$

Integrating with limits at $x=0$ CCL-GDL interface $\eta_{S}=\eta_{S 0}$, and at $\mathrm{x}=1$ CCL-PEM interface $\eta_{S}=\eta_{S 1}$.

$b \int_{\eta_{S_{0}}}^{\eta_{S_{1}}} \exp \left(\eta_{S} / b\right) d \eta_{S}=\frac{R_{P}}{R_{C}} \frac{b j}{j_{0}} \int_{0}^{1} d x \quad$ yields $\quad \exp \left(\eta_{s_{0}} / b\right)=\exp \left(\eta_{s_{1}} / b\right)-\frac{R_{P}}{R_{C}} \frac{j}{j_{0}} \quad$ for $j>j_{0}$

if $R_{P} / R_{C} \geq 1$ therefore $\quad \eta_{s 0} \neq \eta_{s 1}$

but if $R_{P} / R_{C}<<1$ therefore the variation is small $\eta_{s_{0}} \approx \eta_{S_{1}}$ and $\eta_{S}$ can be considered constant along $x$.

\section{List of Symbols}

$b$

tafel slope, $m V$

$c_{O} \quad$ local oxygen concentration through the CCL, $\mathrm{mol} / \mathrm{cm}^{3}$

$c_{O}^{\prime} \quad$ oxygen concentration at the CCL-PEM interface, $\mathrm{mol} / \mathrm{cm}^{3}$

$c_{O}^{*} \quad$ oxygen concentration at the GDL-CCL interface, $\mathrm{mol} / \mathrm{cm}^{3}$

$C_{d l} \quad$ capacitance between dissimilar materials, $\mathrm{F} / \mathrm{cm}^{2}$

D effective diffusion coefficient, $\mathrm{cm}^{2} / \mathrm{seg}$

F $\quad$ faraday constant, $96485 \mathrm{C} / \mathrm{mol}$

i $\quad$ imaginary component in impedance

$j_{o} \quad$ exchange current density, $A / \mathrm{cm}^{2}$

$j_{m} \quad$ maximum rate of ion transfer, $\mathrm{A} / \mathrm{cm}^{2}$

$\bar{j} \quad$ current distribution in catalyst layer, $\mathrm{A} / \mathrm{cm}^{2}$

$L \quad$ inductance of the measurement cables, $H$

$P \quad$ parameter related to CPE (constant phase element)

$R \quad$ ideal gas constant, 8314.3 Joule/mol.K 


$\begin{array}{ll}R_{C} & \text { charge transfer resistance, } \Omega . \mathrm{cm}^{2} \\ R_{e} & \text { total ohmic resistance, PEM, GDL, Plate, } \Omega . \mathrm{cm}^{2} \\ R_{M} & \text { mass transfer resistance, } \Omega . \mathrm{cm}^{2} \\ R_{P} & \text { ionic resistance in cathode catalyst layer, } \Omega . \mathrm{cm}^{2} \\ R_{W} & \text { resistance for the diffusion process, } \Omega . \mathrm{cm}^{2} \\ S & \text { laplace domain } \\ T & \text { temperature, } K \\ T_{W} & \text { time constant for the diffusion process, seg } \\ t & \text { time, seg } \\ V & \text { flux of the oxygen through the CCL, } \mathrm{mol} . \mathrm{cm}^{2} \mathrm{~s} \\ Z & \text { angular frequency, rad/sec } \\ Z & \text { imaginary part of impedance } \\ Z & \text { impedance of cathode catalyst layer, } \Omega . \mathrm{cm}^{2} \\ Z & \text { Warburg impedance, } \Omega . c m^{2} \\ Z & \text { electrons consumed } \\ Z & \end{array}$

\section{Greek}

$\sigma_{P} \quad$ ionic conductivity in the CCL

$\delta \quad$ finite diffusion distance of oxygen transport from CCL-GDL to CCL-PEM interface, $\mathrm{cm}$

$\eta \quad$ overpotential in the CCL, $V$

$\bar{\eta} \quad$ Linearised overpotential in catalyst layer, $V$

$\eta_{S} \quad$ steady state overpotential, $V$ 


\section{Figure Captions}

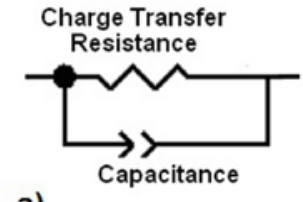

a)

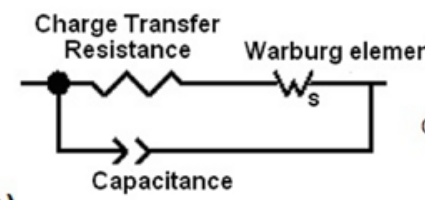

b)

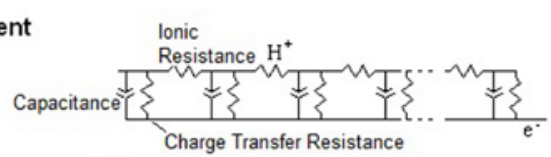

c)

Figure 1 a) Randles circuit for low current operation, b) Randles-Warburg circuit for high current operation, c) Transmission line circuit for low current operation

\section{CCL}

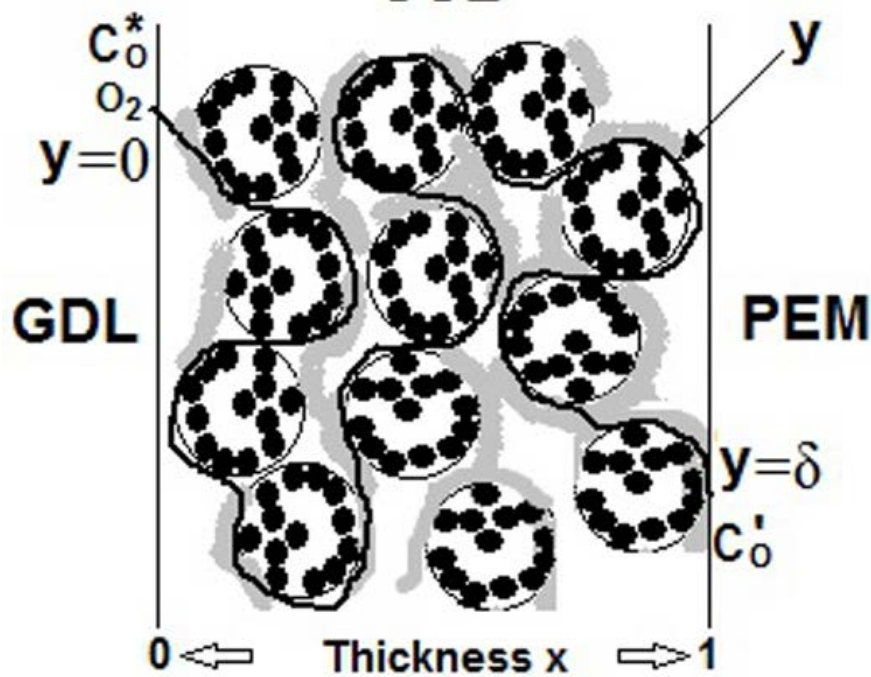

Figure 2 Oxygen transport in the CCL

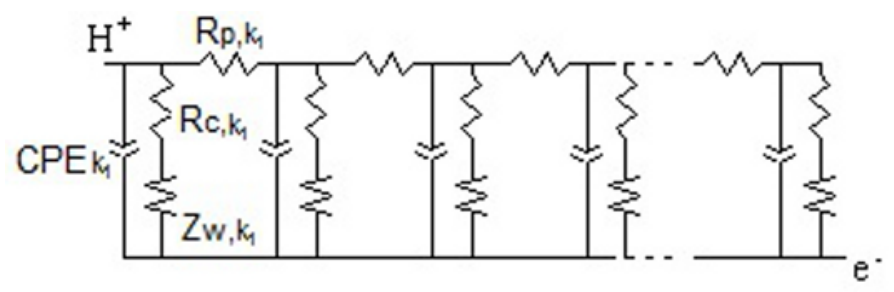

Figure 3 Generic circuit for the CCL characterisation 

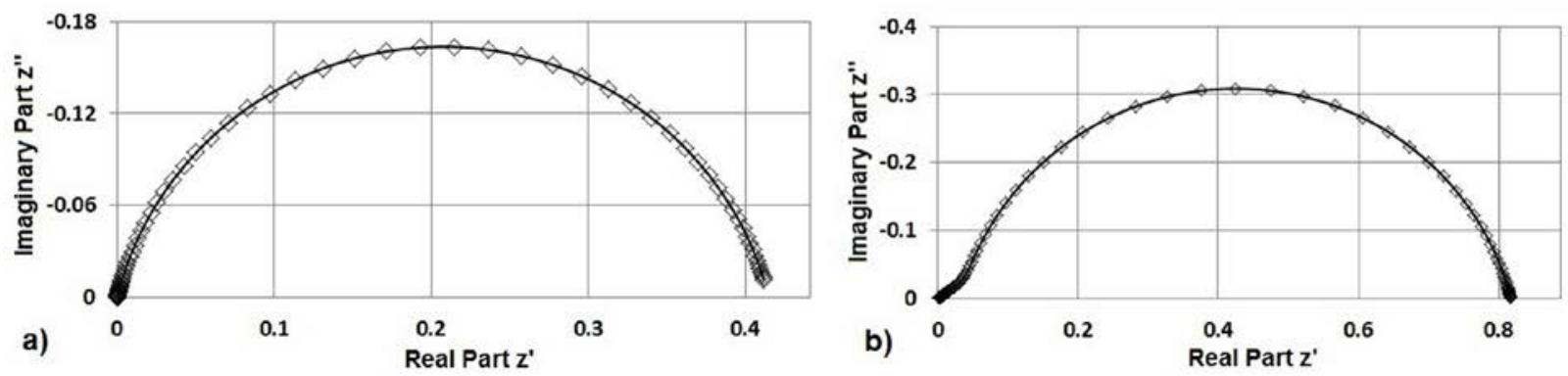

Figure 4 Comparison between circuits considering no oxygen transport limitations $(\diamond)$ and Generic circuit (-), a) Randles, b) Transmission line

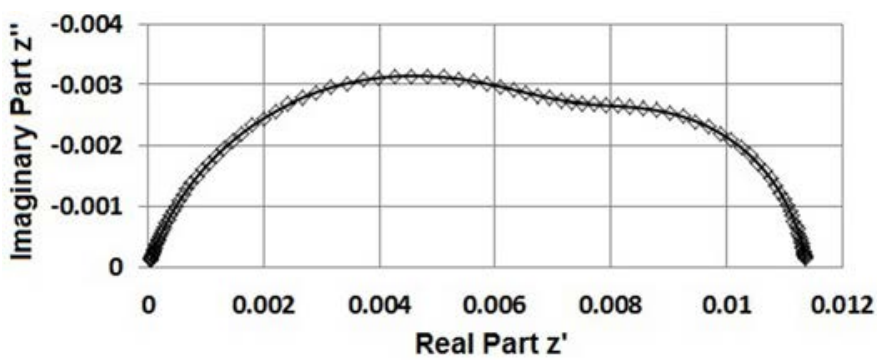

Figure 5 Comparison between Randles-Warburg circuit $(\diamond)$ and Generic circuit (-)

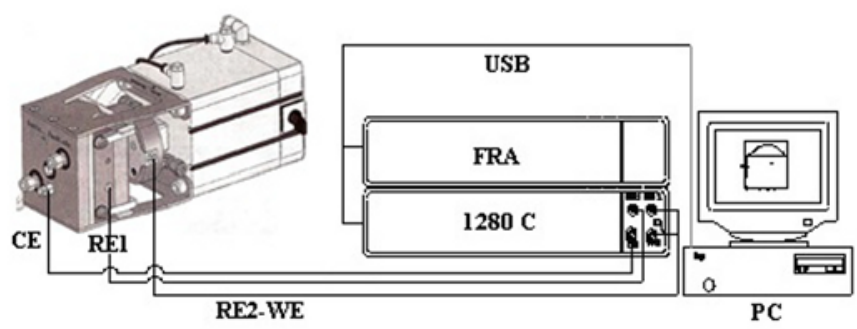

Figure 6 Experimental Set-up
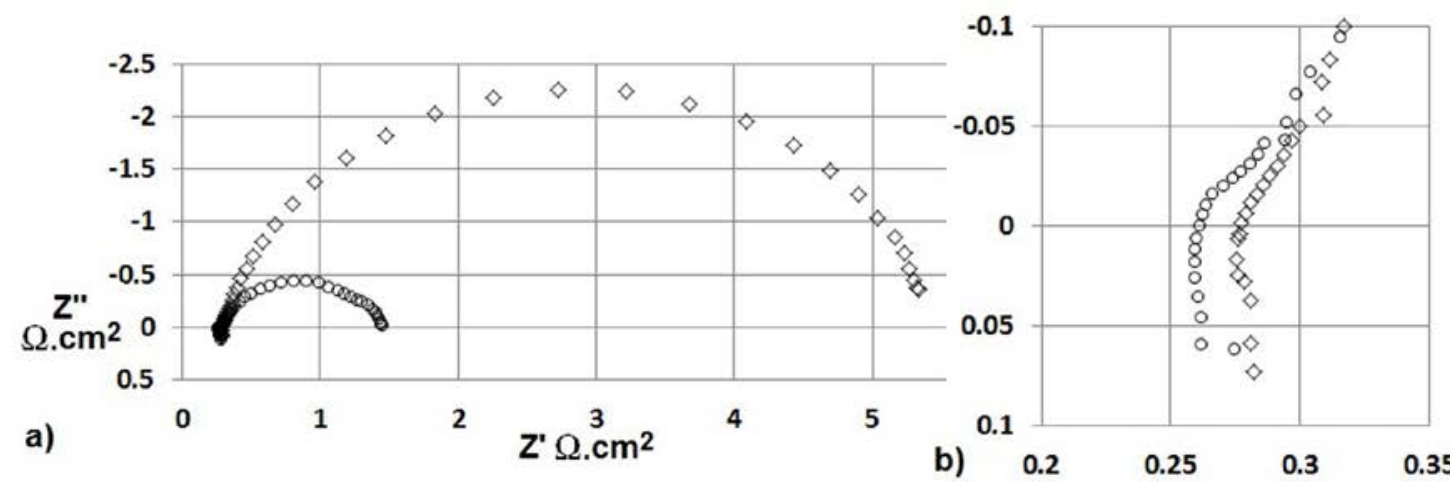

$8 \mathrm{~mA} / \mathrm{cm}^{2}$

$0.12 \mathrm{~A} / \mathrm{cm}^{2}$

Figure 7 Measured Cathode Impedance Response, b) high frequency region 


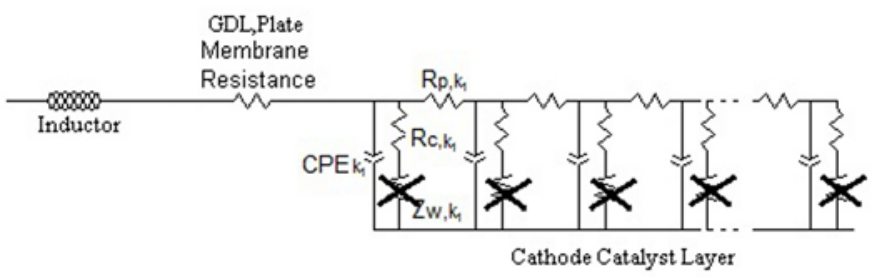

Figure 8 Generic circuit accounting for the cathode neglecting mass transport limitations

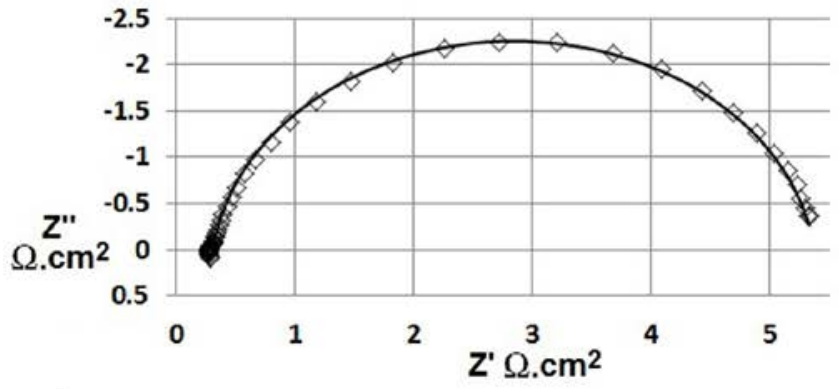

a)

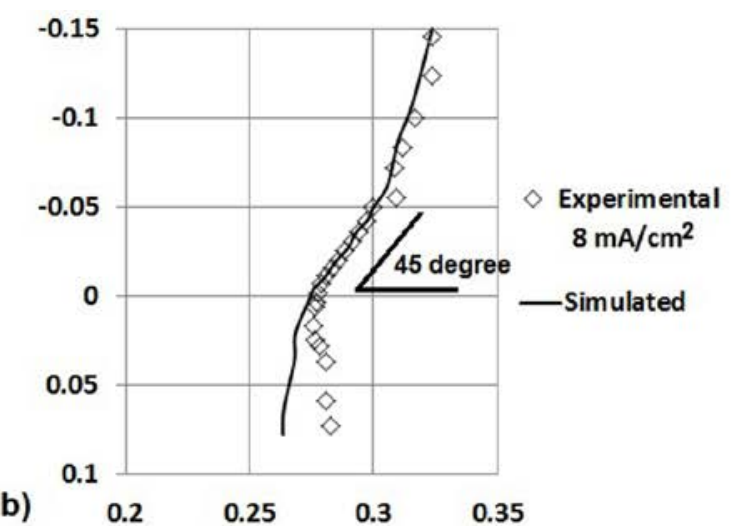

Figure 9 Comparison between simulated (-) and measured $(\diamond)$ data at $0.008 \mathrm{~A} / \mathrm{cm}^{2}$, b) high frequency region

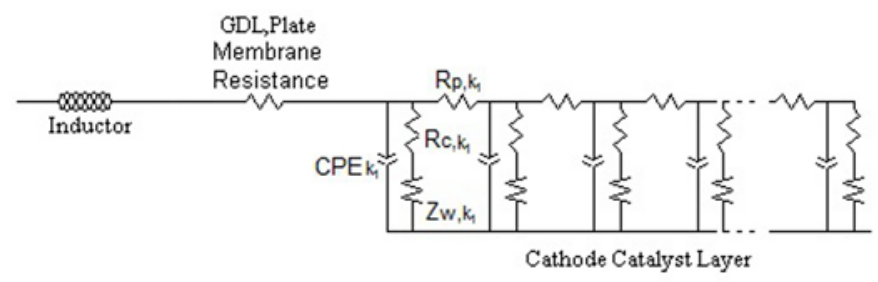

Figure 10 Generic circuit accounting for the cathode considering oxygen transport limitations 


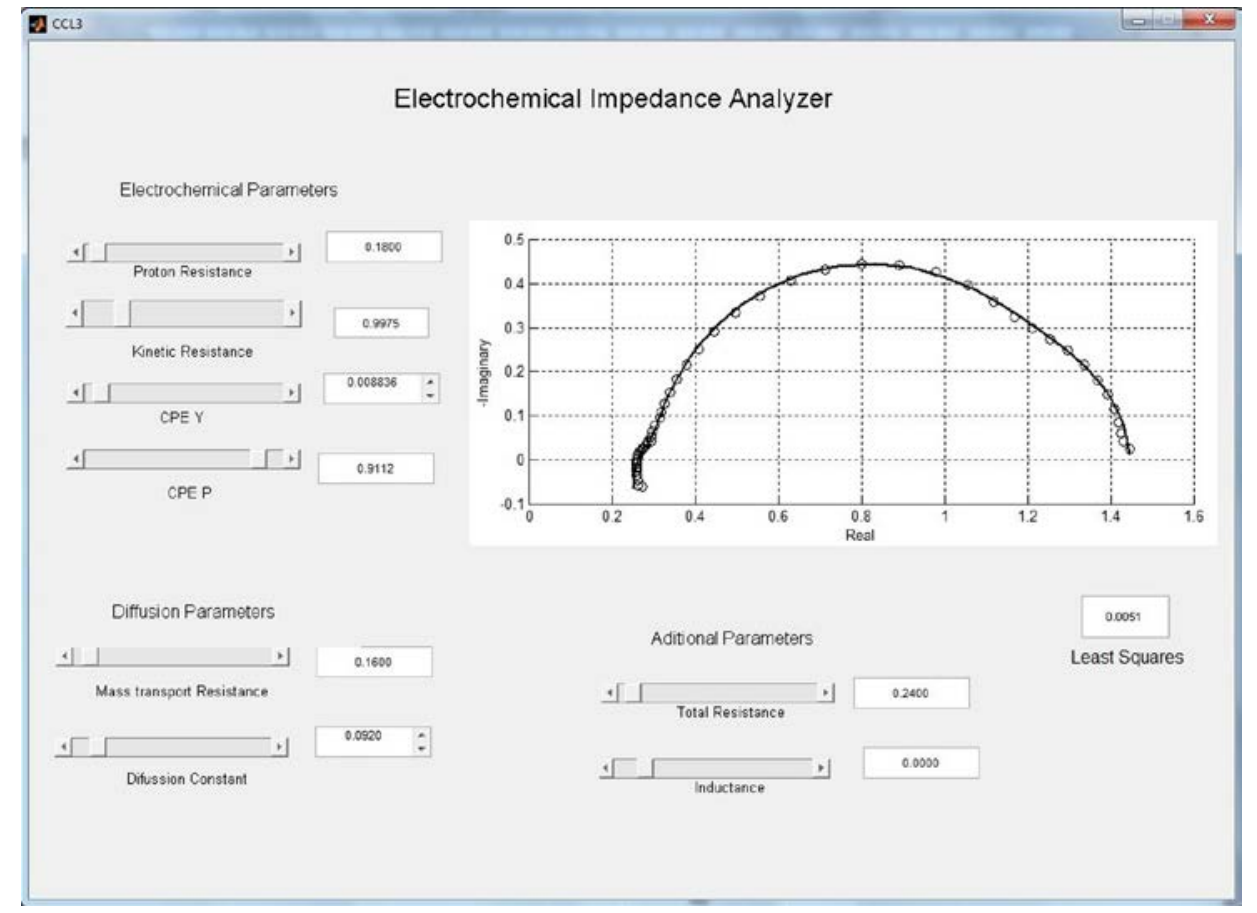

Figure 11 Graphical User Interface for EIS validation
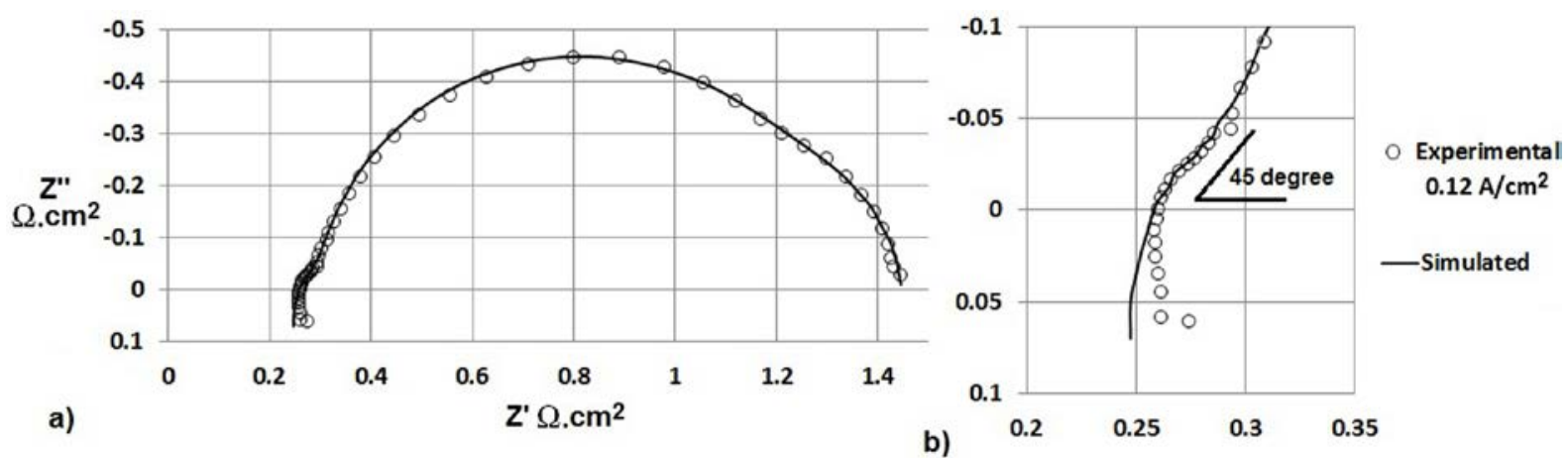

Figure 12 Comparison between simulated (-) and measured (०) data at $0.12 \mathrm{~A} / \mathrm{cm}^{2}$, b) high frequency region 


\section{References}

[1] N. Wagner, E. Gülzow, J. Power Sources 127 (2004) 341-347.

[2] D. Malevich, E. Halliop, B. A. Peppley, J. G. Pharoah, K. Karan, J. Electrochem. Soc. 156 (2009) B216-B224.

[3] M. Ciureanu, R. Roberge, J. Phys. Chem. B 105 (2001) 3531-3539.

[4] N. Fouquet, C. Doulet, C. Nouillant, G. Dauphin-Tanguy, B. Ould-Bouamama, J. Power Sources 159 (2006) 905-913.

[5] M. Eikerling, A. A. Kornyshev, J. Electroanal. Chem. 475 (1999) 107-123.

[6] R. Makharia, M. F. Mathias, D. R. Baker, J. Electrochem. Soc. 152 (2005) A970-A977.

[7] M. C. Lefebvre, R. B. Martin, P. G. Pickup, Electrochem. Solid-State Lett. 2 (1999) 259-261.

[8] T. Suzuki, H. Murata, T. Hatanaka, Y. Morimoto, R\&D Review of Toyota, Toyota Central R\&D Labs, Inc. 39 ( 2003) 33-38.

[9] J. Hou, W. Song, H. Yu, Y. Fu, L. Hao, Z. Shao, B. Yi, J. Power Sources 176 (2008) 118-121.

[10] G. Li, P. G. Pickup, J. Electrochem. Soc. 150 (2003) C745-C752.

[11] N. A. Hampson, S. A. G. R. Karunathilaka, R. Leek, J. of Appl. Electrochem. 10 (1980) 3-11.

[12] M. Ciureanu, H. Wang, J. New Mat. Electrochem. Systems 3 (2000) 107-119.

[13] N. Wagner, J. Appl. Electrochem. 32 (2002) 859-863.

[14] K. Malek, M. Eikerling, Q. Wang, T. Navessin, Z. Liu, J. Phys. Chem. C 111 (2007) 13627-13634.

[15] M. Eikerling, A. A. Kornyshev, J. Electroanal Chem. 453 (1998) 89-106

[16] J. Zhang, PEM Fuel Cell Electrocatalysts and Catalysts Layers, Springer, London, 2008.

[17] C. Berger, Handbook of Fuel Cell Technology, Prentice-Hall, Inc. / Englewood Cliffs, N.J., New York, 1968.

[18] A.A.Kulikovsky, J. Electroanal. Chem, 669 (2012) 28-34.

[19] G. Lin, W. He, T. V. Nguyen, J. Electrochem. Soc. 151 (2004) A1999-A2006.

[20] P. Rama, R. Chen, ASME J. Fuel Cell Sci. Technol. 7 (2010) 051007 - 051023.

[21] D. J. L. Brett, S. Atkins, N. P. Brando, V. Vesovic, N. Vasileiadis, A. Kucernaka, Electrochem. Solid-State Lett. 6 (2003) A63-A66.

[22] C. H. Hsu and F. Mansfeld, Corrosion 57 (2001) 747-748.

[23] A. J. Bard, and L. R Faulkner, Electrochemical Methods, John Wiley \& Sons, Inc., New York, (2001).

[24] V. A. Paganin, C. L. F. Oliveira, E. A. Ticianelli, T. E. Springer and E. R. Gonzalez, Electrochim. Acta 43, (1998) 3761-3766.

[25] W. Mérida, D. A. Harrington, J. M. Le Canut, G. McLean, J. Power Sources 161 (2006) 264-274.

[26] S. Cruz-Manzo, R. Chen and P. Rama, J. Fuel Cell Science Technology, 9 (2012) 051002

[27] S. Cruz-Manzo, R. Chen and P. Rama, Int. J. Hydrogen Energy (2012) http://dx.doi.org/10.1016/j.ijhydene.2012.08.141 